\title{
Desarrollo profesional docente en TIC en contextos interculturales. La experiencia del Bachillerato en Ciencias de la Educación I y II ciclos, con énfasis en Lengua y Cultura Cabécar
}
ICT teacher professional development in intercultural contexts. The experience of the Baccalaureate in Education Sciences, I and II cycles, with an emphasis on language and Cabecar culture
Desenvolvimento profissional de professores de TIC em contextos interculturais. A experiência do Bacharelado em Ciências da Educação I e II ciclos, com ênfase em Língua e Cultura Cabécar

\author{
Jenny Seas Tencio \\ Universidad Estatal a Distancia \\ San José, Costa Rica \\ jseas@uned.ac.cr \\ (1) ORCID: https://orcid.org/0000-0002-6540-9806
}

Recibido - Received - Recebido: 19 / 07 / 2021 Corregido - Revised - Revisado: 19 / 09 / 2021 Aceptado - Accepted - Aprovado: 15 / 10 / 2021

DOI: https://doi.org/10.22458/ie.v23iespecial.3659

URL: https://revistas.uned.ac.cr/index.php/innovaciones/article/view/3659

\begin{abstract}
Resumen: La experiencia del proceso de formación en el uso de las tecnologías de la información y la comunicación (TIC), implementada en el proyecto que imparte la carrera interuniversitaria de bachillerato en Ciencias de la Educación I y II Ciclos, con énfasis en Lengua y Cultura Cabécar, se toma como objeto de estudio para identificar hallazgos que contribuyan en la construcción de una propuesta mejorada y actualizada para la oferta de la tercera cohorte. Se aborda, específicamente, el desarrollo profesional para la adquisición de competencias docentes en el uso de las TIC, el cual ha sido develado, en el Octavo informe del estado de la educación (2021), como una de las áreas deficitarias en el personal docente. La elección de esta área radica en la importancia de que las personas docentes logren un adecuado aprovechamiento de las TIC para el desarrollo de los procesos educativos y el logro de sus metas formativas, con un uso ético, que trascienda en la formación estudiantil. Este análisis se complementa con el estudio de propuestas teóricas y prácticas en torno al desarrollo profesional docente, sin dejar de lado el contexto intercultural del proyecto. Se emplea una metodología cualitativa que parte de la revisión documental. La sistematización permitió definir recomendaciones para la concreción del proceso formativo, la definición de competencias TIC en torno a los procesos de aprender, diseñar, implementar, evaluar e investigar, y un planteamiento estratégico para la ejecución en la tercera cohorte, que se considera factible de aplicar en otras áreas del desarrollo profesional.
\end{abstract}

Palabras clave: Formación docente, competencias del docente, tecnología educacional, educación intercultural, cabécar, desarrollo profesional.

Summary: The experience of the educational process in the use of information and communication technologies (ICT), implemented in the project that imparts the interuniversity career of baccalaureate in Education Sciences I and II Cycles, with an emphasis on Language and Culture Cabécar, is taken as an object of study to identify findings that contribute to the construction of an improved and updated proposal for the offer of the third cohort. It specifically addresses professional development for the acquisition of teaching skills in the use of ICT, which has been revealed, in the Eighth State of Education report (2021), as one of the deficit areas in teaching staff. The choice of this area lies in the importance that teachers achieve an adequate use of ICT for the development of educational processes and the achievement of their educational goals, with ethical use, which transcends in student training. This analysis is complemented by the study of theoretical and practical proposals around teacher professional development, without neglecting the intercultural context of the project. 
A qualitative methodology is used that starts from the documentary review. The systematization allowed defining recommendations for the concretion of the training process, the definition of ICT competencies around the processes of learning, designing, implementing, evaluating and researching, and a strategic approach for the execution in the third cohort, which is considered feasible to apply in other areas of professional development.

Key Words: Teacher training, teacher skills, educational technology, intercultural education, cabécar, professional development.

Resumo: A vivência do processo de formação no uso das tecnologias de informação e comunicação (TIC), implementado no projeto que transmite a carreira interuniversitária de bacharelado em Ciências da Educação I e II Ciclos, com ênfase em Língua e Cultura Cabécar, é tida como um objeto de estudo identificar achados que contribuam para a construção de uma proposta aprimorada e atualizada para a oferta da terceira coorte. Aborda especificamente o desenvolvimento profissional para a aquisição de competências pedagógicas na utilização das TIC, que se revelou, no Oitavo Relatório do Estado da Educação (2021), uma das áreas de défice de pessoal docente. A escolha desta área reside na importância de que os professores alcancem um uso adequado das TIC para o desenvolvimento dos processos educacionais e o alcance dos seus objetivos educacionais, com um uso ético, que transcenda na formação dos alunos. Esta análise é complementada pelo estudo de propostas teóricas e práticas em torno da formação profissional docente, sem descurar o contexto intercultural do projeto. É utilizada uma metodologia qualitativa que parte da revisão documental. A sistematização permitiu definir recomendações para a concretização do processo formativo, a definição de competências TIC em torno dos processos de aprendizagem, conceber, implementar, avaliar e pesquisar, e uma abordagem estratégica para a execução na terceira coorte, considerada viável de aplicação em outras áreas de desenvolvimento profissional.

Palavras chave: Formação de professores, habilidades de professores, tecnologia educacional, educação intercultural, cabécar, desenvolvimento profissional.

\section{INTRODUCCIÓN}

En marzo de 2021, la Comisión de Enlace responsable de la ejecución del proyecto interuniversitario que imparte la carrera de bachillerato en Ciencias de la Educación, I y ll Ciclos, con énfasis en Lengua y Cultura Cabécar (BCELCC), en la que participan la Universidad de Costa Rica (UCR), la Universidad Nacional (UNA) y la Universidad Estatal a Distancia (UNED), todas de Costa Rica, recibió el comunicado por parte del Consejo Nacional de Rectores (CONARE) sobre la aprobación del financiamiento para ofertar una tercera cohorte.

Dicha aprobación dio respuesta a la solicitud planteada por la comunidad cabécar de Chirripó, organizada en torno al Consejo Local de Educación Indígena (CLEI), para continuar con la oferta del plan de estudios, debido a las necesidades de formación docente universitaria con pertinencia cultural que persisten en la zona. Esta aprobación es coherente con el compromiso de las instituciones universitarias públicas costarricenses por la atención de las poblaciones más desfavorecidas a través de acciones afirmativas.

Este nuevo desafío para las universidades involucradas en el proyecto surge en un contexto educativo en crisis, producto de las medidas adoptadas con motivo de la pandemia de la COVID-19, las cuales develaron las grandes asimetrías existentes en el acceso a la educación en modalidades remotas apoyadas por el uso de las tecnologías de la información y la comunicación (TIC); no solo por las limitaciones de acceso a las telecomunicaciones, sino también, entre otros factores, por el limitado dominio en competencias digitales de las personas docentes, evidenciado en la investigación realizada por Zúñiga, Molina, Picado y Solano para el Octavo informe del estado de la educación (2021).

En este contexto, las instituciones de educación superior mantienen su compromiso con la formación docente de calidad y asumen el reto de ofrecer planes de estudios acordes con las necesidades de las poblaciones meta, lo que exige su constante revisión y actualización. En el caso de las carreras de formación docente, considerando los grandes desafíos formativos en el desarrollo de competencias digitales antes descrito, se requiere una respuesta oportuna y pertinente que procure llenar el vacío que existe, con el fin de mejorar las prácticas educativas y, por ende, su calidad. 
En el caso de la carrera de Formación Docente con pertinencia cultural, objeto de estudio de este artículo, y de cara a la oferta de su tercera cohorte, se busca realizar un proceso de mejora a partir del análisis y sistematización de la experiencia desarrollada en las dos cohortes anteriores e incorporar los criterios teóricos y prácticos validados respecto a las competencias que deben ser desarrolladas, desde una óptica de formación continua. Todo ello en busca de la consolidación de procesos educativos especializados para la comunidad indígena cabécar de Chirripó.

La sistematización tiene el propósito de obtener recomendaciones para la gestión de la tercera cohorte que atiendan las necesidades y desafíos formativos con una visión prospectiva. Los aportes podrán ser considerados tanto en espacios de diversidad cultural referida exclusivamente a los pueblos originarios como a la gran gama de escenarios formativos facilitados por la globalización de sociedades conformadas en la actualidad, como efecto de la migración, la exclusión, la diversidad cultural en sus múltiples manifestaciones, entre otros.

\section{DESARROLLO DE LA EXPERIENCIA}

Para describir la experiencia en estudio se parte de ofrecer un retrato del contexto en que se desarrolló, a través de la presentación de datos generales sobre la ubicación geográfica, el contexto educativo en el que fue ejecutado el proyecto de BCELCC y la gestión formativa implementada en torno al uso de las TIC con fines educativos como parte del plan de estudios.

La población meta del proyecto se ubica en el contexto geográfico del territorio indígena cabécar de Chirripó, distrito del cantón de Turrialba. Chirripó se caracteriza por ser una zona de difícil acceso debido a las condiciones de las vías o la inexistencia de estas.

Este grupo humano se encuentra en las posiciones más bajas de las mediciones del desarrollo. Por ejemplo, el último censo realizado en el 2011 arrojó que el 90\% de los hogares de Chirripó tiene al menos una carencia entre los indicadores de condiciones de vida, el 79\% de los hogares posee carencias de vida saludable, el $36 \%$ carencias de acceso al conocimiento y el $71 \%$ carencia de albergue digno (INEC, 2011); condiciones que, a la fecha, no parecen haber mejorado significativamente.

Esta población se ha visto históricamente relegada por los factores estructurales de acceso a la educación y el conocimiento que ofrecen las instituciones del Estado en general, con un ingreso tardío de la escuela y deficientes servicios en cuanto al acceso a las TIC se refiere.

Como se puede constatar en la figura 1, que muestra el mapa de cobertura 3G actualizado el 19 de abril de 2021 para el distrito de Chirripó, se evidencia la cobertura prácticamente inexistente en servicios de telecomunicaciones; y con esta, las nulas posibilidades reales de acceder a la información en la zona y, por consiguiente, las limitaciones para el desarrollo de competencias requeridas para su empleo.

Lo anterior con el agravante de que el idioma de las personas originarias pobladoras (la L1 es el cabécar) ha sido poco estudiado y se tienen limitaciones para regularizar la escritura, que además se ve limitada por la falta de desarrollos informáticos que permitan un uso adecuado en los dispositivos electrónicos y digitales.

Los servicios educativos institucionalizados en el distrito de Chirripó se han expandido en los últimos años con escuelas unidocentes y colegios rurales, que requieren de personal formado para el despeño en contextos interculturales. 
Figura 1. Mapa de cobertura 3G en el cantón de Chirripó.
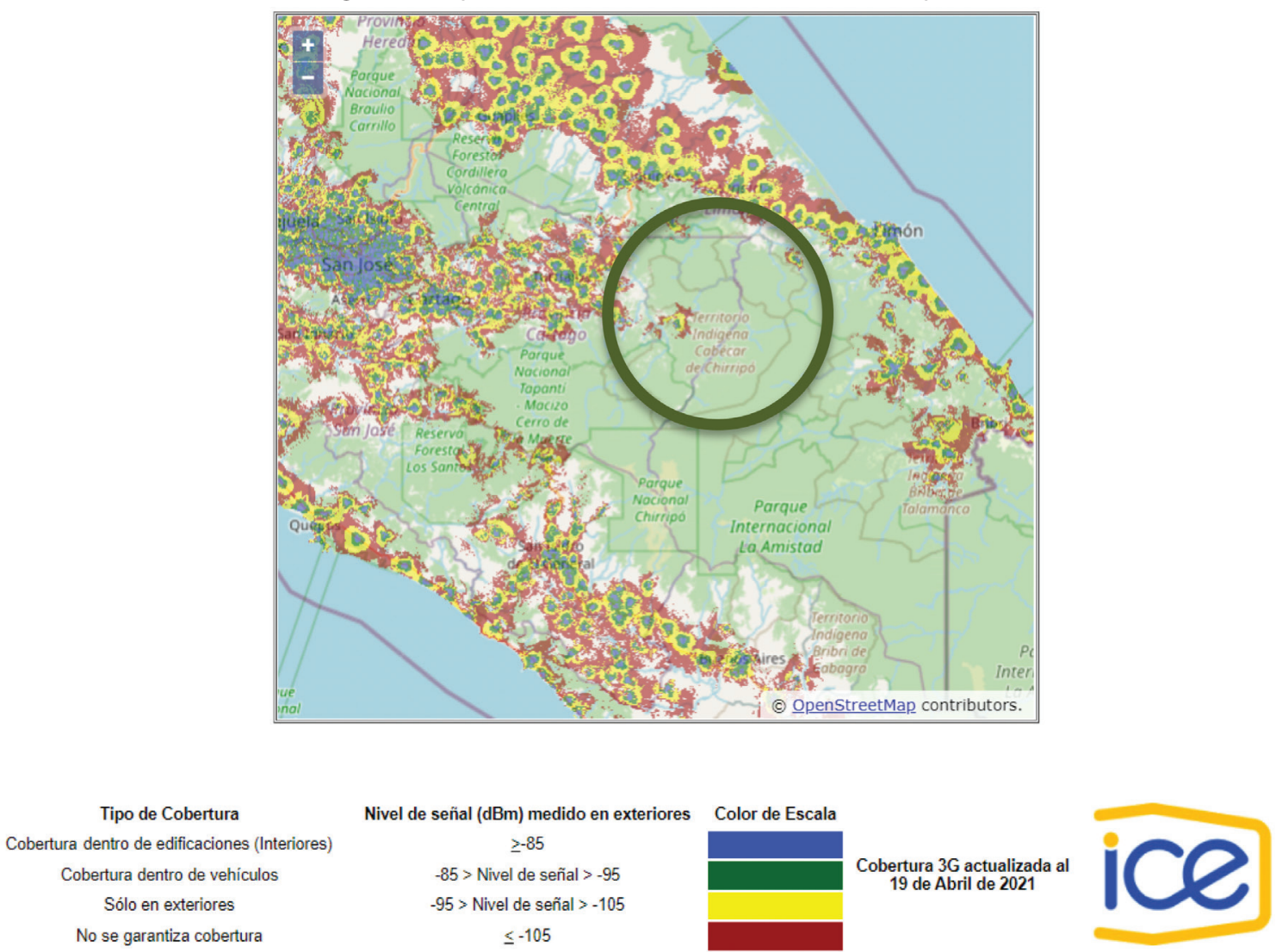

Fuente: ICE (https://mapas.ice.go.cr/MapasCobertura/)

La experiencia se desarrolla en un espacio de convivencia intercultural en el que los elementos que refieren al paisaje cultural, la identidad del territorio, el conocimiento individual y colectivo, las epistemologías y las organizaciones propias del grupo étnico, entre otras, participan e intervienen desde su propia complejidad.

La experiencia fue ejecutada con la oferta de la primera y la segunda cohortes de la carrera de BCELCC, entre los años 2008 al 2019, como parte del proyecto de formación interuniversitaria.

Dicho proyecto se planteó como objetivo general facilitar el acceso a la educación superior de personas indígenas cabécares de Chirripó; para ello, releva la educación como estrategia para el desarrollo integral y autónomo de las comunidades cabécares.

Desde su creación, el plan de estudios se propuso constituirse en una oferta educativa universitaria en correspondencia con los procesos de autonomía y las demandas de las comunidades por una educación de calidad y culturalmente pertinente, que requiere de la actualización constante para garantizar la pertinencia y la calidad de la oferta de cara a una nueva promoción.

La oferta del plan de estudios se realizó bajo convenio específico entre las universidades participantes, con fondos otorgados por el CONARE. La primera cohorte se impartió del 2008 al 2013 y la segunda del 2014 al 2019. La tercera cohorte tiene programada su apertura en el año 2022.

La planificación y la gestión del proceso de desarrollo profesional en el campo de las TIC, que propicia el proyecto, estuvieron compuestas por tres momentos que, para efectos de este análisis, la autora ha 
denominado como: diagnóstico formativo, oferta formativa y capacitación. Esta organización se ilustra en la figura 2 , y se describe seguidamente.

Figura 2. Representación gráfica del proceso de ejecución de la experiencia

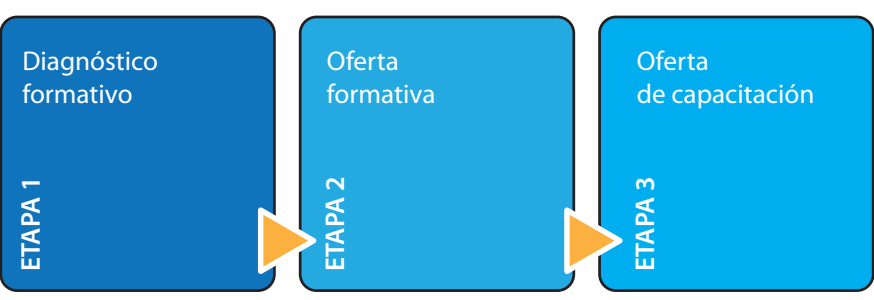

Fuente: Elaboración propia.

De acuerdo con la experiencia, las etapas se caracterizan por:

Etapa I. Diagnóstico formativo: Consistió en la ejecución de un taller sobre aspectos básicos en el uso del procesador de textos de Word y la creación de una cuenta de correo electrónico. Se denomina "diagnóstico participativo" porque las sesiones, además del diagnóstico del nivel de dominio en el uso del equipo computacional permitieron, in situ, la capacitación, la reflexión y la identificación de los requerimientos de las personas, en cuanto a dominios en TIC para su práctica docente.

En esta etapa se identificaron necesidades desde las relativas a conocimientos básicos hasta las habilidades motoras para el uso de hardware. Esta primera etapa aportó información para planificar la oferta educativa de la asignatura de Tecnología Educativa, que es parte de la formación universitaria.

Etapa II. Oferta formativa. La carrera cuenta con una malla curricular compuesta por asignaturas regulares y optativas. Entre las asignaturas optativas se incluye la de Tecnología Educativa, la cual ha sido elegida, históricamente, por todas las personas estudiantes de la carrera.

En la primera cohorte, esta asignatura formó parte del último bloque de estudios, y a solicitud del estudiantado, según se registra en el informe de evaluación, para la segunda cohorte se trasladó al inicio de la formación, justificado en la utilidad de los conocimientos para la carrera y su desempeño laboral.

La oferta incluye encuentros presenciales para el uso de laboratorio y actividades a distancia para el abordaje de aspectos teóricos, acompañados de materiales didácticos mediados. Los encuentros presenciales fueron desarrollados en las sedes de las universidades participantes en el proyecto ubicadas en el cantón de Turrialba, por las razones abordadas en la breve descripción del contexto expuesto.

El objetivo general y los objetivos específicos de la asignatura no tuvieron variación, fueron los mismos tanto para la primera como para la segunda cohorte. En la tabla 1 se presentan el objetivo general y los objetivos específicos planteados.

Como se evidencia en la tabla 1, el objetivo general refiere básicamente al nivel de dominio conceptual y metodológico de la tecnología educativa; y los objetivos específicos detallan los conocimientos aplicados mayoritariamente al uso didáctico de los recursos. No se hace una referencia explícita al desarrollo de competencias en el uso de las TIC, aunque el programa incluye sesiones de laboratorio que permite su abordaje en la práctica. Cabe destacar que se denota la incorporación de procesos que contemplan aspectos culturales y de contextos que le aportan significados al aprendizaje, más allá del dominio de los recursos tecnológicos. 
TABLA 1

Objetivo general y objetivos específicos de la asignatura Tecnología Educativa

\section{OBJETIVO GENERAL}

Analizar diferentes aspectos conceptuales y metodológicos de la tecnología educativa que les permitan a las y los estudiantes reflexionar acerca de las implicaciones que conlleva la incorporación de los procesos y productos tecnológicos en la Educación

\section{OBJETIVOS ESPECÍFICOS}

Adquirir conocimientos acerca de las orientaciones teóricas y metodológicas de la Tecnología Educativa, su conceptualización y aproximación al proceso de mejoramiento de la enseñanza y el aprendizaje.

Determinar los componentes teórico-metodológicos de los principales paradigmas psicopedagógicos, para establecer una visión de la tecnología educativa de acuerdo con cada uno de ellos.

- Discriminar los medios, recursos y productos tecnológicos, su utilización, selección y evaluación desde la práctica educativa.

- Analizar cuáles medios, recursos y productos tecnológicos pueden ser utilizados en la práctica docente, tomado en cuenta el contexto cultural en el que se desempeñan profesionalmente respetando elementos de la diversidad como la lengua, cosmovisión, salud, organización sociopolítica.

- Planear la implementación de medios y recursos para lograr una efectiva integración de la tecnología en el aula contemplando su visión de mundo, patrimonio natural, particularidades históricas y geográficas, condiciones socioeconómicas y su lengua.

- Planificar el desarrollo de contenidos de diversas áreas del currículo utilizando medios y recursos tecnológicos, que permitan estimular valores y actitudes según su contexto cultural y natural.

Fuente: Elaboración propia con base en UCR-UNA-UNED (2016, p. 5-6).

Etapa III. Oferta de capacitación. A partir de la evaluación de la asignatura y los resultados de diagnósticos de necesidades de capacitación, aplicados a las personas graduadas de la carrera, se planifican actividades de seguimiento y capacitación en temáticas de interés para el ejercicio de su profesión.

\section{Andamiaje metodológico para el análisis de la experiencia}

Esta sistematización se propone dar respuesta a la interrogante sobre ¿qué consideraciones deben tomarse en cuenta en la planificación y la gestión del proceso de formación del estudiantado perteneciente a la carrera de bachillerato en Ciencias de la Educación en I y II Ciclos con énfasis en Lengua y Cultura Cabécar, en el uso de las tecnologías de la información y la comunicación con fines educativos, con el propósito de implementarlas en una nueva cohorte?

La intención de fondo surge de la exigencia de diseñar una oferta educativa pertinente, actualizada y de calidad, acorde con el contexto intercultural bilingüe y desde una perspectiva integral de la formación docente.

Para dar respuesta a la pregunta planteada, se emplea una metodología cualitativa que parte de la revisión documental. Los documentos de estudio se generaron en la ejecución de las dos ofertas del plan de estudios realizadas a la fecha, de la oferta de la asignatura de Tecnología Educativa en las mismas cohortes y de las acciones de capacitación emprendidas por el proyecto en el seguimiento de las personas graduadas.

Dichas fuentes están compuestas por: el informe de evaluación externa de la primera cohorte de la carrera, las orientaciones académicas y los informes de ejecución de la oferta de la asignatura de Tecnología Educativa en las dos cohortes impartidas, las evaluaciones realizadas por el estudiantado y el diagnóstico necesidades de capacitación aplicado a las personas graduadas. 
Para abordar el tema de las competencias por desarrollar en la formación docente para el uso de TIC en el ejercicio profesional, se consideran tres instrumentos que son: los estándares ISTE para educadores, la propuesta de formación basada en Competencias y estándares TIC desde la dimensión pedagógica, elaborada por un grupo de investigadores de la Universidad Javeriana con el apoyo de la oficina de la UNESCO en Chile, y el Marco de competencias de los docentes en materia de TIC de la UNESCO. Estos tres instrumentos son de reciente creación y cuentan con organizaciones de prestigio como respaldo académico y metodológico; además, poseen reconocimiento y una amplia difusión en la comunidad académica. Se incorpora al análisis un concepto en construcción, referido a la alfabetización computacional en contextos interculturales, que representa una postura interesante por considerar para este tipo de proyectos como lo es la digiculturalidad.

El análisis de los datos partió de organizar la información extraída de los documentos con la cual identificar las prácticas de planificación y gestión implementadas en la experiencia en torno a la formación docente en TIC, develar las contribuciones del estudiantado y del profesorado -tanto por la práctica pedagógica realizada como sus reflexiones de mejora a futuro- y obtener los hallazgos que mostraran recomendaciones para considerar en la siguiente cohorte.

\section{Análisis de la experiencia}

Como ha sido expuesto, la tercera cohorte de la carrera de BCELCC se desarrollará en un panorama complejo como producto de las medidas adoptadas para enfrentar la pandemia del COVID 19; y que, de acuerdo con la investigación realizada por Zúñiga, Molina, Picado y Solano (2021) para el Octavo informe del estado de la educación, a noviembre de 2020 la evaluación de las competencias digitales de los docentes ubicó a la mayoría en niveles bajos e iniciales de exposición, familiarización y adaptación, con una concentración alta de docentes de primaria y secundaria en niveles iniciales o intermedios en el manejo de TIC. Si se considera el contexto descrito de desarrollo de la experiencia, donde el acceso a las telecomunicaciones y los servicios que lo requieren son bastante limitados, se entiende que las condiciones para la población indígena del proyecto son aún más críticas.

En este contexto se plantea la necesidad de la renovación del proceso formativo en el uso de las TIC, que forma parte del plan de estudios, con el fin de brindar la formación idónea que posibilite su desempeño profesional en contextos interculturales y bilingües.

Al tratarse la experiencia de un proyecto que incursiona en una población indígena, además de la consideración de las nuevas tendencias en el campo de estudio y los nuevos desarrollos tecnológicos aplicados a la educación, se considera de vital importancia tener presente en todo su desarrollo el respeto a la identidad cultural de las personas participantes y sus múltiples manifestaciones, no solo como un mandato ya dispuesto en diferentes normas nacionales e internacionales, sino como un elemento fundamental de los derechos humanos de dicha población y una oportunidad de aprendizaje colectivo para el bien común, lo cual requiere considerar como punto de partida las aspiraciones de la población participante.

De acuerdo con la UNESCO (2019), el desarrollo profesional de las personas docentes debe entenderse como un proceso de aprendizaje durante toda la vida, y no como un evento puntual y único; y aconseja integrar la formación en tres etapas de desarrollo profesional docente que denomina de formación inicial, de formación permanente y de apoyo pedagógico y técnico constante. En todas las etapas se contemplan contenidos relacionados con el uso de la tecnología que incluyen desde el conocimiento de las herramientas y su aplicación pedagógica hasta el apoyo técnico constante, que les permita un uso innovador de las tecnologías y las capacidades para promover un aprendizaje del más alto nivel en el estudiantado. 
Considerando la propuesta desarrollada por el proyecto, se encuentra coherencia en la estructuración de la formación docente con las recomendadas por la UNESCO.

La metodología aplicada parece ser la más idónea, dado que parte de un proceso de indagación y acción, que permite atender necesidades básicas para el aprovechamiento de los recursos tecnológicos, su abordaje en el plan de estudios aplicado a la pedagogía, el desarrollo curricular y la contextualización intercultural.

La definición de los objetivos, las capacidades o competencias, según sea el modelo educativo, y los contenidos de estudio, deben partir de las grandes aspiraciones por alcanzar por parte del cuerpo docente en su contexto laboral. Ambos requieren de la actualización constante, dado que si algo caracteriza a las tecnologías es su pronta obsolescencia, con nuevos desarrollos cada vez más especializados.

Durante la oferta, el estudiantado entra en contacto con diferentes tecnologías de la información y la comunicación y sus posibilidades para construir ambientes de aprendizaje; planea la implementación de medios y recursos en el aula y su aplicación en diversas áreas del currículo.

En cuanto a los contenidos, se encuentra los de índole teórica y práctica, que incluyen aspectos tecnológicos a través de la planificación y el diseño de recursos didácticos con TIC, abordados de manera progresiva en las diferentes etapas.

Para abordar este tema desde la mirada prospectiva, se toman como referencia algunos estándares para el uso de las tecnologías por parte de las personas docentes, ampliamente reconocidos, y las posturas sobre la digiculturalización.

Desde lo expuesto, se considera oportuno visualizar el proceso formativo del desarrollo profesional tal como lo plantea la UNESCO, considerando el contexto de la experiencia y los fines de mejora continua; por ello, se integran al proceso las estrategias de planificación y evaluación con sustento en la indagación teórica y la gestión, y el contexto intercultural como eje central, tal como se representa en la figura 3.

Figura 3. Representación gráfica del proceso de ejecución de la experiencia

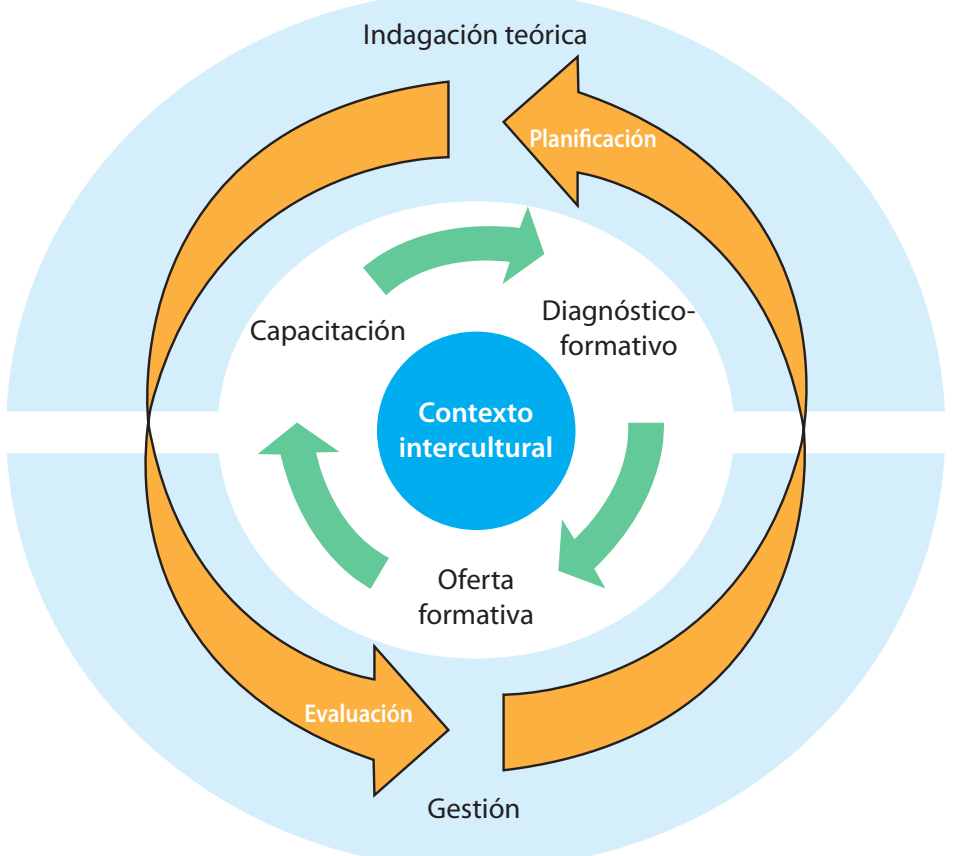

Fuente: Elaboración propia. 
Como se puede notar en la figura 3, la forma de las flechas quiere representar un proceso constante de indagación y acción que permitan recoger información de cada etapa para emplearla como insumo en hacer los ajustes requeridos por la siguiente.

Se suma la gestión académica del proceso formativo que incorpora lo que la autora denomina "indagación teórica", referente a los ámbitos pedagógicos, culturales, curriculares y de tecnología educativa con los cuales problematizar y mejorar la práctica universitaria; así como la gestión administrativa, institucional y comunitaria requerida para ejecutar las acciones propuestas. Estos últimos aspectos se amplían más adelante como resultado del análisis y en la definición de los contenidos para el desarrollo de las competencias digitales.

Por otro lado, de los documentos examinados se pudieron obtener recomendaciones de mejora proporcionadas por parte de docentes, estudiantes y Comisión de Enlace. Estos insumos se recopilan en la figura 4.

Figura 4. Elementos sugeridos para la mejora identificados para cada etapa.

Requerimientos en la organización del tiempo.

Organizar la capacitación para grupos de 15 personas.

Ofrecer atención indivualizada y para grupos de 3 personas máximo.

Desarrollar competencias digitales de forma transversal.

Ejecución de actividades prácticas diversas.

Andamiaje personalizado.

Desarrollar un programa propedéutico para el desarrollo de competencias digitles.

Establecer la asignatura como obligatoria y ubicarla en ciclos iniciales del plan de estudios.

Incorporar el tema en ofertas de capacitación de ciclos interlectivos.

Ejecución de actividades prácticas diversas.

Andamiaje personalizado.

Diagnosticar requerimientos de capacitación aplicables en la labor profesional.

Ofrecer capacitación en uso de recursos novedosos para el abordaje de los contenidos de los planes de estudio.

Monitoreo permanente de las necesidades de capacitación.

Ejecución de actividades prácticas diversas.

Andamiaje personalizado.

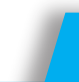

\section{Fase I}

Fase II

Como se puede notar, dentro de los aspectos sugeridos para la mejora se destaca el desarrollo de competencias digitales de manera transversal en todas las etapas, lo cual es coherente con la necesidad de actualización del programa en esta materia.

Las pautas respecto a las capacidades que deban alcanzar las personas docentes en su formación para un mejor desempeño laboral se han definido tradicionalmente a través de los denominados estándares para la formación docente, y los referidos a las TIC no son la excepción. La razón, como señala la ISTE (International Society for Technology in Education), obedece a que la incorporación de estas herramientas en la capacitación y desarrollo de planes de estudios al día de hoy asegura que los futuros maestros estén listos para usar la tecnología con el propósito de profundizar en el aprendizaje de los estudiantes, capaces de adaptarse al uso de una amplia variedad de tecnologías para diversas situaciones y contextos, y preparados para enseñar y liderar dentro de las aulas y escuelas de hoy y de mañana. 
La definición de las competencias o requerimientos formativos en TIC que deben considerarse en el proceso formativo parte del estudio de los tres instrumentos (uno de la ISTE y dos de UNESCO) que ya se han mencionado y se describirán brevemente más adelante.

Asimismo, para contextualizar esos instrumentos desde un enfoque etnoeducativo, acorde con las condiciones de interculturalidad del proyecto, el abordaje deberá partir del conocimiento, el análisis, la valoración y la afirmación de la identidad étnica del grupo cabécar de Chirripó, con la participación de las personas indígenas y las redes interculturales que se establecen y denominan con el constructo emergente de digiculturalidad.

La ISTE es una organización no lucrativa integrada por personas líderes a nivel mundial en el área de tecnología educativa. Conforman una red abierta para guiar el uso eficaz de la tecnología en la educación y la formación del profesorado. ISTE ha establecido un conjunto de estándares ampliamente aceptados y una comunidad colaborativa para su alcance.

De acuerdo con lo que informa en su página web, sus estándares proporcionan las competencias para aprender, enseñar y liderar en la era digital por medio de una hoja de ruta integral para el uso efectivo de la tecnología en las escuelas de todo el mundo. Basados en la investigación de la ciencia del aprendizaje y en la experiencia de los profesionales, los estándares ISTE aseguran que el uso de la tecnología puede crear experiencias de aprendizaje de alto impacto, sostenibles, escalables y equitativas. Asimismo, en su última versión incorporan los planteamientos de la Agenda 2030 para el Desarrollo Sostenible.

Esta organización plantea estándares para estudiantes, maestros, líderes educativos, entrenadores y maestros. En el caso de las personas docentes, certifica siete niveles que van del básico al experto, los cuales denomina "aprendiz", "líder", "ciudadano", "colaborador", "diseñador", "facilitador" y "analista", y para cada uno define las competencias que se resumen en la tabla 2.

TABLA 2

Estándares ISTE para educadores

\begin{tabular}{c|l} 
Aprendiz & $\begin{array}{l}\text { Competencia } \\
\text { Los educadores mejoran continuamente su práctica dependiendo de y con otros, y explorando prácticas } \\
\text { probadas y prometedoras que aprovechan la tecnología para mejorar el aprendizaje de los estudiantes. }\end{array}$ \\
\hline Líder & $\begin{array}{l}\text { Los educadores buscan oportunidades de liderazgo para apoyar el emprendimiento y el éxito de los estu- } \\
\text { diantes en la mejora de la enseñanza y el aprendizaje. }\end{array}$ \\
\hline Ciudadano & $\begin{array}{l}\text { Los educadores inspiran a los estudiantes a contribuir positivamente y para participar responsablemente } \\
\text { en el mundo digital. }\end{array}$ \\
\hline Diseñador & $\begin{array}{l}\text { Los educadores dedican tiempo a colaborar con colegas y estudiantes para mejorar la práctica, descubrir y } \\
\text { compartir recursos e ideas y resolver problemas. } \\
\text { adaptan a la variabilidad del alumno. }\end{array}$ \\
\hline Facilitador & $\begin{array}{l}\text { Los educadores facilitan el aprendizaje con tecnologías para apoyar el logro estudiantil de los estándares } \\
\text { ISTE para estudiantes. }\end{array}$ \\
\hline Analista & $\begin{array}{l}\text { Los educadores comprenden y utilizan los datos para impulsar su construcción y ayudar a los estudiantes a } \\
\text { lograr sus objetivos de aprendizaje. }\end{array}$ \\
\hline & \begin{tabular}{l} 
Fuente: Elaborada a partir de https://www.iste.org/standards/iste-standards-for-teachers \\
\hline
\end{tabular}
\end{tabular}

Los criterios que contemplan los estándares incluyen los relativos a: a) tecnología, operatividad y conceptos, b) planeamiento y diseño de ambientes y experiencias de aprendizaje, c) enseñanza, aprendizaje y currículo, d) valoración y evaluación con TIC, e) uso de tecnología para la productividad, y f) uso ético, legal y equitativo de las TIC. 
Otra propuesta considerada se deriva del estudio de Competencias y estándares TIC desde la dimensión pedagógica, elaborado por la Universidad Javeriana y la UNESCO, el cual privilegia las competencias relacionadas con el diseño, la implementación y la evaluación de espacios educativos mediados por TIC. De acuerdo con los resultados del estudio, las competencias en el diseño de escenarios educativos apoyados en TIC se refieren a las habilidades de planificación y organización de elementos que permitan la construcción de competencias y estándares TIC desde la dimensión pedagógica. Las competencias relacionadas con la implementación dan cuenta de las habilidades que permiten poner en marcha el diseño y planificación de un escenario educativo, y que se ven reflejadas en las prácticas educativas de un docente. Las competencias de evaluación de la efectividad de los escenarios educativos apoyados en TIC se relacionan con las habilidades que le permiten al docente valorar la efectividad para favorecer el aprendizaje significativo en los estudiantes, al incorporar las TIC a sus prácticas educativas (p.16-17).

Estos tres grupos de competencias se organizan en tres niveles de apropiación que denominan "integración", "reorientación" y "evolución". En el nivel de integración las personas docentes utilizan las TIC como una herramienta para presentar contenidos, comunicarse y transmitir información. En el nivel de reorientación se contemplan las prácticas educativas que facilitan la construcción de conocimiento. Y en el nivel de evolución las prácticas de innovación "generan dinámicas impensables sin la presencia de las $\mathrm{TIC}^{\prime \prime}$ (p. 16). El tabla 3 presenta una síntesis de esta propuesta.

TABLA 3

Marco de competencias de los docentes en materia de TIC

\begin{tabular}{|c|c|c|c|c|}
\hline \multicolumn{2}{|c|}{ Competencia } & Nivel integración & Nivel reorientación & Nivel evolución \\
\hline Diseña & $\begin{array}{l}\text { Diseña escenarios } \\
\text { educativos apoyados } \\
\text { en TIC para el apren- } \\
\text { dizaje significativo y la } \\
\text { formación integral del } \\
\text { estudiante. }\end{array}$ & $\begin{array}{l}\text { Conoce que las TIC apor- } \\
\text { tan al almacenamiento, } \\
\text { comunicación, transmi- } \\
\text { sión e intercambio de in- } \\
\text { formación en el diseño de } \\
\text { un escenario educativo. }\end{array}$ & $\begin{array}{l}\text { Organiza estratégicamen- } \\
\text { te el uso de las TIC para } \\
\text { el almacenamiento de } \\
\text { información durante el } \\
\text { diseño de un escenario } \\
\text { educativo. }\end{array}$ & $\begin{array}{l}\text { Modifica adaptativamen- } \\
\text { te la organización del uso } \\
\text { de las TIC para el almace- } \\
\text { namiento de información } \\
\text { durante el diseño de un } \\
\text { escenario educativo. }\end{array}$ \\
\hline Implementa & $\begin{array}{l}\text { Implementa experien- } \\
\text { cias de aprendizaje } \\
\text { significativo apoyadas } \\
\text { en TIC. }\end{array}$ & $\begin{array}{l}\text { Conoce cómo implemen- } \\
\text { tar las TIC para el almace- } \\
\text { namiento, comunicación, } \\
\text { trasmisión e intercambio } \\
\text { de información en un es- } \\
\text { cenario educativo. }\end{array}$ & $\begin{array}{l}\text { Utiliza las TIC para el al- } \\
\text { macenamiento, comu- } \\
\text { nicación, transmisión } \\
\text { e intercambio de infor- } \\
\text { mación en un escenario } \\
\text { educativo. }\end{array}$ & $\begin{array}{l}\text { Modifica adaptativamen- } \\
\text { te el uso de las TIC para } \\
\text { el almacenamiento, co- } \\
\text { municación, transmisión } \\
\text { e intercambio de infor- } \\
\text { mación en un escenario } \\
\text { educativo. }\end{array}$ \\
\hline Evalúa & $\begin{array}{l}\text { Evalúa la efectividad } \\
\text { de los escenarios edu- } \\
\text { cativos apoyados en } \\
\text { TIC para favorecer el } \\
\text { aprendizaje significati- } \\
\text { vo de los estudiantes. }\end{array}$ & $\begin{array}{l}\text { Conoce que las TIC faci- } \\
\text { litan la evaluación de la } \\
\text { efectividad con relación } \\
\text { al almacenamiento, co- } \\
\text { municación, transmisión } \\
\text { e intercambio de infor- } \\
\text { mación en un escenario } \\
\text { educativo. }\end{array}$ & $\begin{array}{l}\text { Utiliza las TIC para evaluar } \\
\text { la efectividad con relación } \\
\text { al almacenamiento, co- } \\
\text { municación, transmisión } \\
\text { e intercambio de infor- } \\
\text { mación en un escenario } \\
\text { educativo. }\end{array}$ & $\begin{array}{l}\text { Modifica adaptativamen- } \\
\text { te el uso de las TIC para } \\
\text { evaluar la efectividad con } \\
\text { relación al almacenamien- } \\
\text { to, comunicación, trans- } \\
\text { misión e intercambio de } \\
\text { información en un esce- } \\
\text { nario educativo. }\end{array}$ \\
\hline
\end{tabular}

Fuente: Elaborado a partir de UNESCO, 2019.

Un tercer instrumento es el Marco de competencias de los docentes en materia de TIC, de la UNESCO (2019), en su tercera versión. De acuerdo con el documento de referencia, "está ideado para preservar las competencias que siguen siendo pertinentes y enmarcarlas en el contexto de los actuales avances tecnológicos y las exigencias cambiantes de la vida y el trabajo" (p. 5). Sobre las tecnologías, incluyen los recursos educativos abiertos (REA) y la educación inclusiva. Una síntesis del marco se presenta en el tabla 4. 
TABLA 4

Marco de competencias de los docentes en materia de TIC

\begin{tabular}{|c|c|c|c|}
\hline Aspectos & $\begin{array}{l}\text { Adquisición de } \\
\text { conocimientos }\end{array}$ & $\begin{array}{l}\text { Profundización de } \\
\text { conocimientos }\end{array}$ & $\begin{array}{l}\text { Creación de } \\
\text { conocimientos }\end{array}$ \\
\hline $\begin{array}{l}\text { Comprensión del } \\
\text { papel de las TIC en las } \\
\text { políticas educativas }\end{array}$ & $\begin{array}{l}\text { Determinar cómo y en qué } \\
\text { medida sus prácticas do- } \\
\text { centes se corresponden con } \\
\text { las políticas institucionales } \\
\text { y/o nacionales y apoyan su } \\
\text { consecución. }\end{array}$ & $\begin{array}{l}\text { Idear, modificar y aplicar prácticas } \\
\text { docentes que contribuyen a la con- } \\
\text { secución de políticas nacionales y/o } \\
\text { institucionales, compromisos inter- } \\
\text { nacionales, por ejemplo convenios } \\
\text { de las Naciones Unidas, y priorida- } \\
\text { des sociales. }\end{array}$ & $\begin{array}{l}\text { Efectuar una reflexión crítica } \\
\text { acerca de las políticas educati- } \\
\text { vas tanto institucionales como } \\
\text { nacionales, proponer modifi- } \\
\text { caciones, idear mejoras y an- } \\
\text { ticipar los posibles efectos de } \\
\text { dichos cambios. }\end{array}$ \\
\hline $\begin{array}{l}\text { Currículoy } \\
\text { evaluación }\end{array}$ & $\begin{array}{l}\text { Analizar las normas cu- } \\
\text { rriculares y determinar el } \\
\text { posible uso pedagógico } \\
\text { de las TIC para cumplir sus } \\
\text { normas. }\end{array}$ & $\begin{array}{l}\text { Integrar las TIC de forma transversal } \\
\text { en los contenidos disciplinares, los } \\
\text { procesos de enseñanza y evaluación } \\
\text { y niveles de curso, y crear un entor- } \\
\text { no de aprendizaje potenciado por } \\
\text { las TIC en el cual los alumnos, con la } \\
\text { ayuda de estas tecnologías, cumplan } \\
\text { con las normas y niveles curriculares. }\end{array}$ & $\begin{array}{l}\text { Determinar las modalidades } \\
\text { óptimas de un aprendizaje co- } \\
\text { laborativo y centrado en el edu- } \\
\text { cando, con miras a alcanzar los } \\
\text { niveles requeridos por currícu- } \\
\text { los multidisciplinarios. }\end{array}$ \\
\hline Pedagogía & $\begin{array}{l}\text { Seleccionar adecuadamen- } \\
\text { te las TIC en apoyo a me- } \\
\text { todologías de enseñanza y } \\
\text { aprendizaje. }\end{array}$ & $\begin{array}{l}\text { Diseñar actividades de aprendizaje } \\
\text { basadas en proyectos y apoyadas } \\
\text { por las TIC, y utilizar las TIC para ayu- } \\
\text { dar a los alumnos a crear, aplicar y } \\
\text { seguir planes de proyecto, y resolver } \\
\text { problemas complejos. }\end{array}$ & $\begin{array}{l}\text { Al determinar los parámetros } \\
\text { del aprendizaje, promover la } \\
\text { autogestión de los alumnos } \\
\text { en el marco de un aprendizaje } \\
\text { colaborativo y centrado en el } \\
\text { educando. }\end{array}$ \\
\hline $\begin{array}{l}\text { Aplicación de } \\
\text { competencias }\end{array}$ & $\begin{array}{l}\text { Conocer las funciones de } \\
\text { los componentes de equi- } \\
\text { pos informáticos y los pro- } \\
\text { gramas de productividad } \\
\text { más comunes, y ser capaz } \\
\text { de utilizarlos. }\end{array}$ & $\begin{array}{l}\text { Combinar diversos recursos y he- } \\
\text { rramientas digitales integrados de } \\
\text { aprendizaje, para ayudar a los alum- } \\
\text { nos a desarrollar capacidades de re- } \\
\text { solución de problemas y de reflexión } \\
\text { de alto nivel. }\end{array}$ & $\begin{array}{l}\text { Construir comunidades del } \\
\text { conocimiento y utilizar herra- } \\
\text { mientas digitales para promo- } \\
\text { ver el aprendizaje permanente. }\end{array}$ \\
\hline $\begin{array}{l}\text { Organización y } \\
\text { administración }\end{array}$ & $\begin{array}{l}\text { Organizar el entorno físico } \\
\text { de tal manera que la tec- } \\
\text { nología esté al servicio de } \\
\text { distintas metodologías de } \\
\text { forma inclusiva. }\end{array}$ & $\begin{array}{l}\text { Utilizar herramientas digitales de } \\
\text { forma flexible, para facilitar el apren- } \\
\text { dizaje colaborativo, gestionar a los } \\
\text { alumnos y a otras partes involucra- } \\
\text { das en el aprendizaje, y administrar } \\
\text { el proceso de aprendizaje. }\end{array}$ & $\begin{array}{l}\text { Liderar la elaboración de una } \\
\text { estrategia tecnológica para } \\
\text { la escuela, a fin de conver- } \\
\text { tirla en una organización de } \\
\text { aprendizaje. }\end{array}$ \\
\hline $\begin{array}{l}\text { Aprendizaje profesio- } \\
\text { nal de los docentes }\end{array}$ & $\begin{array}{l}\text { Utilizar las TIC para su pro- } \\
\text { pio perfeccionamiento } \\
\text { profesional. }\end{array}$ & $\begin{array}{l}\text { Utilizar la tecnología para interac- } \\
\text { tuar con redes profesionales con } \\
\text { miras a potenciar su propio perfec- } \\
\text { cionamiento profesional. }\end{array}$ & $\begin{array}{l}\text { Desarrollar, experimentar, in- } \\
\text { novar y compartir prácticas óp- } \\
\text { timas de forma continua, para } \\
\text { determinar de qué manera la } \\
\text { tecnología puede presentar los } \\
\text { mejores servicios de la escuela. }\end{array}$ \\
\hline
\end{tabular}

Fuente: Elaborado a partir de UNESCO, 2019.

En referencia al contexto interculturalidad y el uso de las TIC, el constructo de digiculturalidad emerge con fuerza a través de plataformas y comunidades virtuales que son, a la vez, camino y destino de una conciencia comunitaria intercultural donde lo importante es crecer individual y socialmente, valorando positivamente la diversidad cultural y los valores universalmente aceptados contemplados en los derechos humanos (Martínez, 2010).

Se concibe como una competencia digital de conocimiento y valoración de la diversidad cultural a través de las TIC. Leiva y Priegue (2012), a partir del estudio de diversos autores, identifican como características conceptuales de la digicultura las siguientes: 
- Necesidad de multialfabetizar al estudiantado para que sepa adaptarse a los diferentes cambios y necesidades de la sociedad, y a la característica de desarrollo y aplicabilidad práctica en red, apoyado en las dimensiones del aprendizaje definidas por Martínez (2010) como: instrumental, para adquirir las habilidades instrumentales para buscar información y uso de tecnologías; cognitiva, para saber plantear problemas, analizar e interpretar la información; sociocomunicacional, que refiere a las habilidades para crear documentos, difundirlos e interaccionar, y axiológica, basada en el desarrollo de actitudes, valores y prácticas sociales éticas. Desarrollo y aplicabilidad práctica en red generado a partir de valores de cooperación e interacción cultural democrática.

En el proceso educativo la persona docente toma el bagaje de conocimientos culturales y de los conocidos como universales, y los sitúa en la experiencia cotidiana del estudiantado; para ello, debe dar respuesta a dos interrogantes básicas: ¿qué se enseña? y ¿cómo se enseña?

En respuesta a la primera interrogante, la experiencia devela la importancia de partir de la reivindicación de los elementos tecnológicos propios de la cultura y las formas de transmisión del conocimiento, para posteriormente abordar los elementos tecnológicos requeridos para la cotidianidad. Sobre la segunda interrogante, se consideran las técnicas de mediación pedagógica utilizadas por la persona docente para potenciar el aprendizaje, los recursos didácticos, la distribución y uso del tiempo en el espacio de aprendizaje y el desarrollo de un currículum que potencie la concreción de capacidades y oportunidades en un contexto identificado con grandes carencias formativas.

En el caso de la experiencia en estudio, las técnicas reportadas como de mayor éxito fueron la mediación explicativa y demostrativa, y la mediación con recursos virtuales. En relación con los recursos, se aprovecharon los laboratorios institucionales y los dispositivos telefónicos inteligentes propiedad del estudiantado, y recursos simbólicos impresos, digitales y audiovisuales. Las estrategias de formación a distancia con la construcción y participación en comunidades de aprendizaje en entornos de aprendizaje móviles. La implementación de técnicas enfocadas en el desarrollo de productos digitales de forma práctica se consideró la forma más adecuada de evaluación en la asignatura y su contexto, porque privilegia la presentación de evidencias a través de aplicar los contenidos abordados en las sesiones de trabajo.

En el análisis de los cuatro instrumentos descritos se encuentra una serie de aspectos comunes que pueden ser considerados en los procesos de formación en TIC, como el que se plantea en este artículo, dado el consenso que existe entre ellos. Entre las coincidencias más notorias se determina que:

- Dimensionan el desarrollo profesional como un proceso a lo largo de la vida. Las propuestas coinciden en que el desarrollo profesional no se adscribe a procesos de formación o capacitación de forma aislada; por el contrario, se concibe de forma permanente a lo largo de la vida.

- Identifican niveles de dominio progresivo de los recursos TIC. Por ejemplo, la Universidad Javeriana los organiza desde el nivel de adquisición de conocimientos, le sigue la profundización y culmina con el nivel experto en la creación de contenidos. La UNESCO, por su parte, los distingue en los niveles de integración, orientación y evolución, y el ISTE define siete niveles que van del aprendiz al analista. Considerar esos niveles supone desprenderse de una organización basada en el estudio de recursos o software específico para centrarlos en estrategias de dominio. En síntesis, se focalizan en estándares para la formación permanente que van desde un estado inicial a uno superior, con una visión progresiva de dominio.

- Articulan las competencias tecnológicas con las pedagógicas. Esta integración hace que los procesos formativos tengan sentido por su aplicación en el ejercicio profesional. Algunos ejemplos que evidencian esta articulación son: entre los estándares de la UNESCO se propone Analizar las normas curriculares y determinar el posible uso pedagógico de las TIC para cumplir sus normas; en los estándares ISTE: Los educadores comprenden y utilizan los datos para impulsar su construcción y ayudar a los 
estudiantes a lograr sus objetivos de aprendizaje, y del estudio de la Universidad Javeriana: Implementa experiencias de aprendizaje significativo apoyadas en TIC. En síntesis, el proceso formativo se liga con el quehacer profesional docente.

- Involucran situaciones de aprendizaje de autogestión y de relación con alumnos y colegas. Se plantea un desarrollo profesional ligado al accionar docente en el ambiente de aprendizaje y en la comunidad educativa, así como para su propio crecimiento profesional. Se consideran espacios individuales y de interacción social para el aprendizaje, con lo que se fortalece la comunicación y el desarrollo colectivo que potencia incluso procesos de innovación y vivencia profesional.

- Vinculan los conocimientos y el uso de las TIC entre docentes y estudiantes. Esta vinculación se presenta desde la digicultaridad, en la que se contempla la necesidad de multialfabetizar al estudiantado; desde la propuesta de UNESCO, la posibilidad de utilizar herramientas digitales para ayudar a los alumnos a desarrollar capacidades de resolución de problemas y de reflexión de alto nivel, y la propuesta de la Universidad Javeriana de diseñar escenarios educativos apoyados en TIC para el aprendizaje significativo y la formación integral del estudiante.

A partir del análisis de los marcos de competencias y estándares considerados, se identifican cuatro dimensiones o grandes ejes por considerar en las futuras propuestas de desarrollo profesional en TIC, que se recopilan en la figura 5 .

Figura 5. Dimensiones o áreas de competencia para la formación docente en el uso de TIC

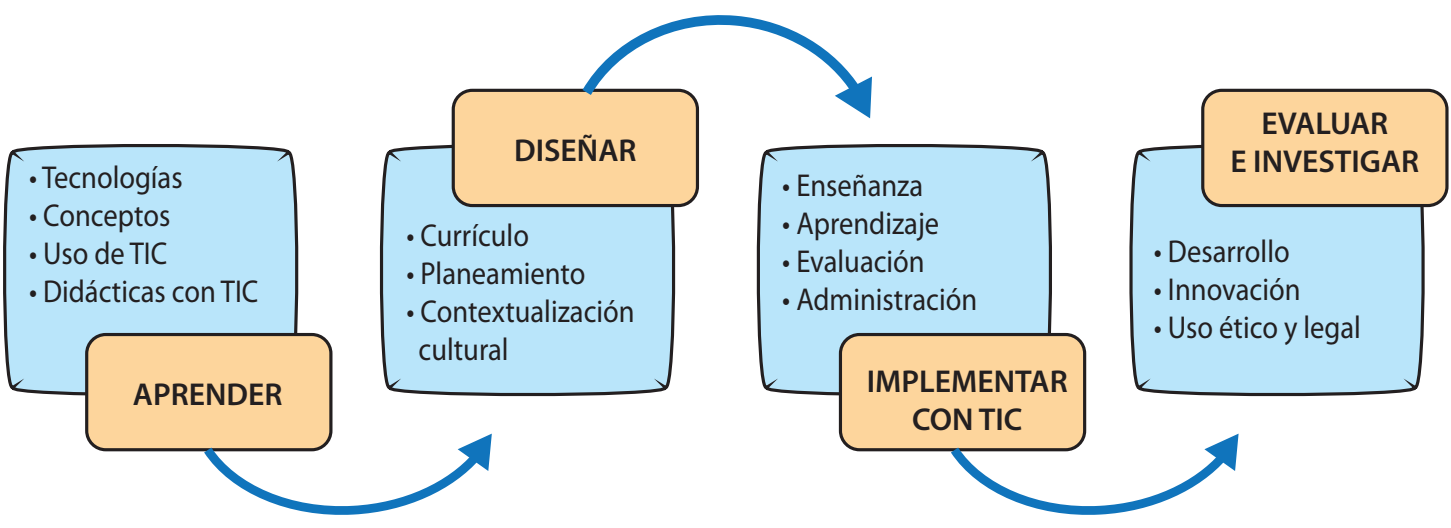

Fuente: Elaboración propia.

La primera dimensión, identificada como Aprender, se relaciona con el aprendizaje básico en el uso de herramientas TIC e incorpora su uso con fines didácticos. La segunda involucra el uso de los recursos en el contexto educativo y se prevén desde los instrumentos de planificación, por lo que se denomina Diseñar. La tercera dimensión se denomina Implementar con TIC, que corresponde al ejercicio profesional. Y la última, que supone un uso más especializado de las TIC, a partir de la rigurosidad investigativa en la solución de problemas y la innovación con TIC, se identifica como Evaluar e Investigar. 


\section{SÍNTESIS Y REFLEXIONES FINALES}

Como reflexiones finales de la experiencia, a partir del análisis esbozado y en respuesta a la interrogante planteada, se identifican aspectos por considerar o implementar en los procesos de actualización y formulación de la oferta formativa de carácter intercultural, para el uso de las TIC con fines educativos.

- Una educación pertinente en el siglo XXI no puede diseñarse sin incluir el entorno tecnológico que forma parte de la cultura contemporánea, como recurso que ofrece grandes oportunidades para acceder al conocimiento, sin alejarse de los saberes compartidos por el colectivo más cercano.

- El ámbito de formación docente que enmarca el proyecto promueve un uso de las tecnologías de la información y la comunicación con sentido; es decir, estrechamente ligado a los propósitos sociales, de conocimiento y de comunicación. Esta perspectiva es crucial de mantener en la planificación y ejecución de la tercera cohorte, dadas las posibilidades de otorgar significado al aprendizaje.

- Se considera oportuno visualizar el proceso formativo del desarrollo profesional en TIC, tal como lo plantea la UNESCO, como un proceso de aprendizaje durante toda la vida. La estructuración del proceso realizado por el proyecto de BCELCC en las etapas identificadas como diagnóstico formativo, oferta formativa y capacitación es coherente con lo recomendado por la UNESCO, por lo que su implementación de cara a la formulación de la tercera es pertinente.

- La necesidad imperante de atender y reconocer las concepciones culturales, las formas de transmisión de conocimientos y el respeto a las epistemologías propias de los individuos y sus colectivos son cruciales en la actualidad, en tiempos en que las opciones y espacios interculturales son cada vez más frecuentes y enriquecedores de los espacios de formación compartidos.

- La vinculación de los conocimientos de estudio y los elementos tecnológicos culturales con el uso de las TIC entre docentes y estudiantes es necesaria para potenciar los aprendizajes, los recursos didácticos, la distribución y uso del tiempo en el espacio de aprendizaje por medio de las técnicas consideradas más exitosas por el profesorado, como la mediación explicativa y demostrativa.

- Aprender, Diseñar, Implementar, Investigar y Evaluar, con compromiso ético, son las grandes áreas de competencia que se deben contemplar en la actualidad, en un programa o proyecto de formación y desarrollo profesional docente con tecnologías de la información y la comunicación, con un abordaje ligado a la práctica docente y la interacción profesional en los espacios de aprendizaje.

\section{REFERENCIAS}

Programa Estado de la Nación (2021). Octavo informe del estado de la educación: resumen. San José: PEN-CONARE.

Instituto Costarricense de Electricidad (ICE) (s.f.). https://mapas.ice.go.cr/MapasCobertura/

Instituto Nacional de Estadística y Censos (INEC) (2011). Distribución porcentual de las personas por número de carencias críticas, según cantón. Censo 2011. http://www.inec.go.cr/censos/censos-2011

International Society for Technology in Education (ISTE) (s.f.). https://www.iste.org/standards/ iste-standards-for-teachers

Leiva, J.J. y Priegue, D. (2012). Educación intercultural y TIC: claves pedagógicas de la innovación y el cambio social en el siglo XXI. @TIC: Revista d'Innovació educativa, (9), 32-43. https://dialnet.unirioja.es/ servlet/articulo?codigo $=4294298$ 
Martínez, F. (2010). Las redes digitales como marco para la multiculturalidad. Sevilla: MAD Eduforma.

UCR-UNA-UNED (2009). Plan de estudios de Bachillerato en Ciencias de la Educación, I y II Ciclos, con énfasis en Lengua y Cultura Cabécar. CONARE.

UCR-UNA-UNED (2016). Orientaciones para el curso Tecnología Educativa.

UNESCO (2019). Marco de competencias de los docentes en materia de TIC. http://eduteka.icesi.edu.co/pdfdir/unesco-competencias-tic-docentes-version-3-2019.pdf

Valencia-Molina, T., Serna-Collazos, A., Ochoa-Angrino, S., Caicedo-Tamayo, A., Montes-González, J. \& Chávez-Vescance, J. (2016). Competencias y estándares TIC desde la dimensión pedagógica: Una perspectiva desde los niveles de apropiación de las TIC en la práctica educativa docente. Pontificia Universidad Javeriana, Cali. http://www.unesco.org/new/fileadmin/MULTIMEDIA/FIELD/Santiago/pdf/Competencias-estandaresTIC.pdf

Zúñiga, M., Molina, M. I., Picado, K. y Solano, R. (2021). Competencias digitales de los docentes: desafíos y ruta de acción para lograr un uso efectivo y sostenido de las TIC al servicio del mejoramiento educativo. Ponencia preparada para el Octavo informe del estado de la educación. San José: PEN-CONARE. 